\title{
Making temperature-insensitive optofluidic photonic-crystal devices
}

\author{
Christian Karnutsch, Ross McPhedran, and \\ Benjamin J. Eggleton
}

Infiltrating the air holes of a lattice with liquid enables construction of thermally stable resonant devices.

Resonant optical cavities are major building blocks in many applications, from microlasers and high-sensitivity biomedical sensor systems to optical filters, switches, and integrated circuits. Device performance depends strongly on their stability against changes in ambient conditions. For example, refractive-index sensors based on optical-resonance techniques suffer from temperature drift that introduces noise and degrades sensitivity. ${ }^{1}$ As a second example, the thermal stability of integrated photonic devices is crucial for making commercially viable integrated optoelectronic circuits.

Recently, several techniques have been proposed to create temperature-insensitive nanophotonic devices. One group micromachined a Fabry-Pérot cavity into a single-mode optical fiber. $^{2}$ A second team employed a microstructured fiber containing a Bragg grating and infiltrated its holes using a refractiveindex material with a negative thermooptic coefficient. ${ }^{3}$ Other approaches include silicon-on-insulator waveguides coated with negative-coefficient polymer cladding ${ }^{4}$ and silicon waveguides in a balanced Mach-Zehnder configuration. ${ }^{5}$ We suggest a novel approach that is adaptable to a wide range of applications. ${ }^{6}$ It is based on the tendency of fluid refractive indices to decrease with temperature, while those of a range of dielectrics vary in the opposite way. Thus, with an appropriate design, these opposing tendencies cancel each other out, creating a thermally balanced optical configuration.

The key concept of our scheme relies on infiltrating the lattice with a liquid that has a negative thermooptic coefficient, which balances the positive thermal drift of the host photoniccrystal (PhC) material. Figure 1 shows the temperature dependence of the refractive indices for silicon and a commercially

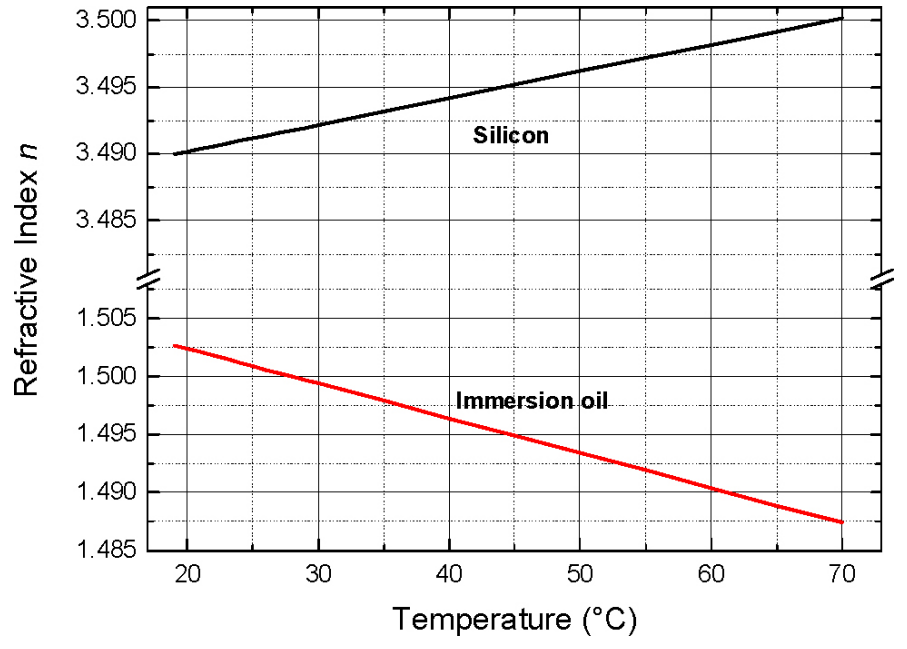

Figure 1. Refractive index versus temperature for silicon and a liquid at a wavelength of $\approx 1410 \mathrm{~nm}$.

available Cargille immersion oil, type B (with thermooptic coefficients $\partial n_{\mathrm{Si}} / \partial T=+2 \times 10^{-4} / \mathrm{K}$ for the former and $\partial n_{\mathrm{L}} / \partial T=$ $-3 \times 10^{-4} / \mathrm{K}$ for the latter). For PhC waveguides with air holes penetrated by liquid, the effective refractive index experienced by guided modes depends on the combination of the two (host material and liquid), weighted by the filling fraction $f$. This represents the relative electric-field overlap with the corresponding material. PhC host materials (silicon and III-V-type semiconductors) have positive coefficients, ${ }^{7}$ as opposed to most liquids ${ }^{8}$ and polymers. ${ }^{9}$ Therefore, a large range of pairings has an effective combined index that renders devices temperature insensitive if the guided mode has an appropriate fraction of electric-field overlap in each material.

To form a microfluidic double-heterostructure cavity (see Figure 2), ${ }^{10-12}$ we infiltrated Cargille immersion oil (see Figure 3) into-silicon $\mathrm{PhC}$ membranes. Figure 4 shows transmission 


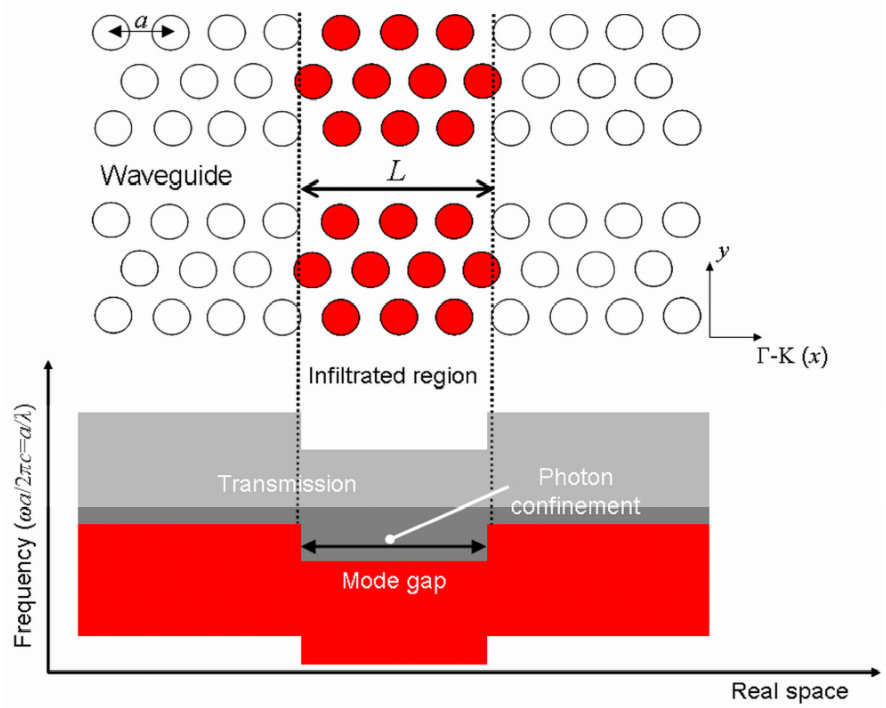

Figure 2. Schematic of the optofluidic double-heterostructure cavity. (top) Photonic-crystal slab containing a line defect. Fluid penetrating the air holes leads to a mode-gap effect. (bottom) Band diagram along the waveguide direction. The transmission region (gray) allows photon propagation in the waveguide, which is suppressed by the mode-gap section (red). Photons with frequencies within the mode gap can only propagate in the infiltrated waveguide region. a: Lattice period of the crystal. L: infiltrated cavity length. $\omega$ : Frequency. c: Speed of light. $\lambda$ : Wavelength. $\Gamma-K(x)$ : Direction of symmetry in the crystal lattice.

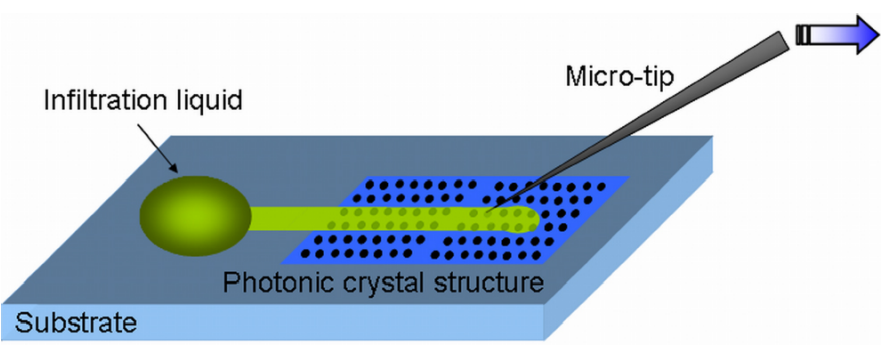

Figure 3. Infiltration process. We immerse a microtip into a liquid and draw it across a photonic crystal to create a microfluidic cavity.

spectra as a function of temperature while probing an infiltrated optofluidic cavity. The microfluidic cavity sustained Fabry-Pérot resonances with moderate quality factors of order 15,000-20,000.

We found that the resonance wavelengths of the Fabry-Pérot cavity shift with temperature (see Figure 4). In the temperature range investigated, resonances (2) to (5) show a blue shift between -0.03 (2) and $-0.06 \mathrm{~nm} / \mathrm{K}(5)$, while (1) remains exceptionally stable at $\lambda=1405 \mathrm{~nm}$, with an extremely low gradient of $-0.003 \mathrm{~nm} / \mathrm{K}$. This represents a 20 -fold reduction in temperature sensitivity compared to (5) and a 27-fold decrease compared

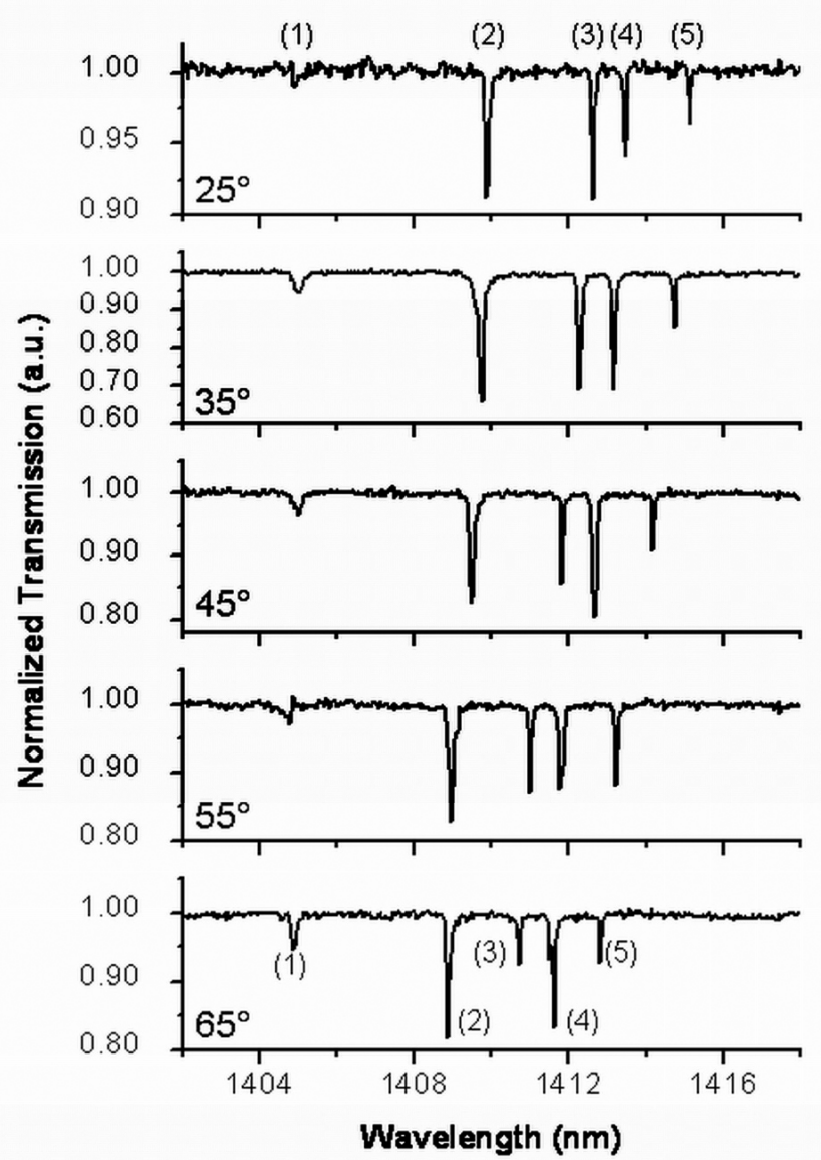

Figure 4. Transmission spectra at various temperatures measured on an optofluidic cavity of length $6.8 \mu \mathrm{m}$. (1)-(5) indicate the resonance wavelengths. a.u.: Arbitrary units.

to a standard silicon-PhC waveguide. ${ }^{13}$ The data in Figure 4 demonstrates an important generic property: if a system of elements with opposing temperature dependencies creates a range of resonances, then there will often be one where field sharing leads to temperature insensitivity.

In summary, we have demonstrated a scheme to make nanophotonic devices independent of their environment's temperature. This promotes development of robust, high-sensitivity sensor systems that respond to liquid refractive-index changes, while reducing the complexity caused by thermal fluctuations. Temperature insensitivity may also pave the way for highprecision nanophotonic components such as microlasers, filters, and switches. Future work will incorporate thermally stable cavities into fully integrated, reconfigurable optical circuits. We plan

Continued on next page 
to combine numerous optofluidic components on the same platform through selective air-hole infiltration, enabling highly complex optical capabilities.

We thank the Australian Research Council (ARC) for generous support. We acknowledge collaborations with students and colleagues on various aspects of optofluidic cavities, in particular with Cameron $L$. C. Smith, Alexandra Graham, Snjezana Tomljenovic-Hanic, Christian Grillet, Christelle Monat, Sanshui Xiao, N. Asger Mortensen, Liam O'Faolain, and Thomas F. Krauss.

\section{Author Information}

\section{Christian Karnutsch}

School of Physics

Institute of Photonics and Optical Science (IPOS)

University of Sydney

Sydney, Australia

http:/ / www.physics.usyd.edu.au/cudos/people/

ckarnutsch.htm

Christian Karnutsch heads the optofluidics and plasmonics research group at the Center for Ultrahigh-bandwidth Devices for Optical Systems (CUDOS). His research interests include optofluidic PhC devices, nanophotonics, and organic and inorganic semiconductor lasers. From September 2009 he will be a professor of optical sensor systems at the University of Applied Sciences in Karlsruhe, Germany. He has contributed many papers to SPIE conferences, mainly on optofluidics, nanotechnology, and organic and III-V-compound semiconductor lasers, sensors, and LEDs.

\section{Ross McPhedran and Benjamin J. Eggleton}

School of Physics

IPOS

University of Sydney

Sydney, Australia

http:/ / www.usyd.edu.au/ipos/

Ross McPhedran is a professorial research fellow at CUDOS. His research interests include $\mathrm{PhC}$, transport properties of composite materials, theory of microstructured fibers, diffraction gratings, plasmonics, and methods of mathematical physics.

Benjamin Eggleton is an ARC federation fellow and professor of physics, as well as director of CUDOS. His research includes nonlinear optics, PhC fibers, optical signal processing, and optofluidics.

\section{References}

1. I. M. White and X. Fan, On the performance quantification of resonant refractive index sensors, Opt. Express 16 (2), pp. 1020-1028, 2008. doi:10.1364/OE.16.001020

2. T. Wei, Y. Han, Y. Li, H.-L. Tsai, and H. Xiao, Temperature-insensitive miniaturized fiber inline Fabry-Perot interferometer for highly sensitive refractive index measurement, Opt. Express 16 (8), pp. 5764-5769, 2008. doi:10.1364/OE.16.005764

3. N. Mothe, D. Pagnoux, M. C. Phan Huy, V. Dewinter, G. Laffont, and P. Ferdinand, Thermal wavelength stabilization of Bragg gratings photowritten in holefilled microstructured optical fibers, Opt. Express 16 (23), pp. 19018-19033, 2008 doi:10.1364/OE.16.019018

4. W. N. Ye, J. Michel, and L. C. Kimerling, Athermal high-index-contrast waveguide design, IEEE Photon. Technol. Lett. 20 (11), pp. 885-887, 2008. doi:10.1109/LPT.2008.922338

5. A. Densmore, D. X. Xu, S. Janz, P. Waldron, T. Mischki, G. Lopinski, A. Delâge, J. Lapointe, P. Cheben, B. Lamontagne, and J. H. Schmid, Spiral-path high-sensitivity silicon photonic wire molecular sensor with temperature-independent response, Opt. Lett. 33 (6), pp. 596-598, 2008. doi:10.1364/OL.33.000596

6. C. Karnutsch, C. L. C. Smith, A. Graham, S. Tomljenovic-Hanic, R. McPhedran, B. J. Eggleton, L. O'Faolain, T. F. Krauss, S. Xiao, and N. A. Mortensen, Temperature stabilization of optofluidic photonic crystal cavities, Appl. Phys. Lett. 94 (23), pp. 231114-3, 2009. doi:10.1063/1.3152998

7. F. G. Della Corte, G. Cocorullo, M. Iodice, and I. Rendina, Temperature dependence of the thermo-optic coefficient of InP, GaAs, and SiC from room temperature to $600 \mathrm{~K}$ at the wavelength of 1.5 $\mu \mathrm{m}$, Appl. Phys. Lett. 77 (11), pp. 1614-1616, 2000.

8. C. B. Kim and C. B. Su, Measurement of the refractive index of liquids at 1.3 and 1.5 micron using a fibre optic Fresnel ratio meter, Meas. Sci. Technol. 15 (9), pp. 1683-1686, 2004.

9. Z. Zhang, P. Zhao, P. Lin, and F. Sun, Thermo-optic coefficients of polymers for optical waveguide applications, Polym. 47 (14), pp. 4893-4896, 2006.

10. C. Grillet, C. Monat, C. L. C. Smith, M. W. Lee, S. Tomljenovic-Hanic, C. Karnutsch, and B. J. Eggleton, Reconfigurable photonic crystal circuits, Laser Photon. Rev., 2009.

11. C. L. C. Smith, U. Bog, S. Tomljenovic-Hanic, M. W. Lee, D. K. Wu, L. O'Faolain, C. Monat, C. Grillet, T. F. Krauss, C. Karnutsch, R. C. McPhedran, and B. J. Eggleton, Reconfigurable microfluidic photonic crystal slab cavities, Opt. Express 16 (20), pp. 15887-15896, 2008. doi:10.1364/OE.16.015887

12. U. Bog, C. L. C. Smith, M. W. Lee, S. Tomljenovic-Hanic, C. Grillet, C. Monat, L. O'Faolain, C. Karnutsch, T. F. Krauss, R. C. McPhedran, and B. J. Eggleton, High-Q microfluidic cavities in silicon-based two-dimensional photonic crystal structures, Opt. Lett. 33 (19), pp. 2206-2208, 2008. doi:10.1364/OL.33.002206

13. M. Uenuma and T. Moooka, Temperature-independent silicon waveguide optical filter, Opt. Lett. 34 (5), pp. 599-601, 2009. doi:10.1364/OL.34.000599 